The Homogenization and Optimization of Thermoelectric Composites

Jiangyu Li

UNIVERSITY OF WASHINGTON

04/17/2015

Final Report

DISTRIBUTION A: Distribution approved for public release. 


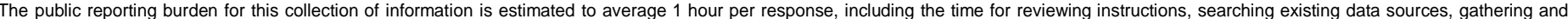

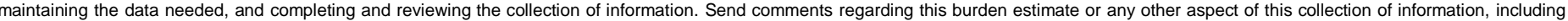

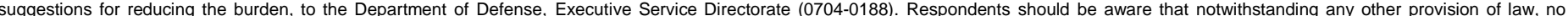
person shall be subject to any penalty for failing to comply with a collection of information if it does not display a currently valid OMB control number.

PLEASE DO NOT RETURN YOUR FORM TO THE ABOVE ORGANIZATION.
1. REPORT DATE $(D D-M M-Y Y Y Y)$ 2. REPORT TYPE
$4 / 12 / 2015$
Final Report
3. DATES COVERED (From - To)

\section{TITLE AND SUBTITLE}

The Homogenization and Optimization of Thermoelectric Composites

5b. GRANT NUMBER

FA9550-12-1-0325

5c. PROGRAM ELEMENT NUMBER

6. AUTHOR(S)

Jiangyu Li

\section{5d. PROJECT NUMBER}

5e. TASK NUMBER

5f. WORK UNIT NUMBER

\section{PERFORMING ORGANIZATION NAME(S) AND ADDRESS(ES)}

University of Washington

Seattle, WA 98195-2600

\section{SPONSORING/MONITORING AGENCY NAME(S) AND ADDRESS(ES)}

AF Office of Scientific Research

875 N. Randolph St. Room 3112

Arlington, VA 22203
8. PERFORMING ORGANIZATION REPORT NUMBER

\section{DISTRIBUTION/AVAILABILITYSTATEMENT}

DISTRIBUTION A

\section{SUPPLEMENTARY NOTES}

\section{ABSTRACT}

We seek to understand the effective behavior of thermoelectric composites using rigorous homogenization technique in this project. In the last three years, our accomplishment includes: (1) rigorous analysis of thermoelectric field distribution and effective properties of layered thermoelectric structures, showing that their effective figure of merit is not bounded by the constituent phases, in contrast to the previous claim; (2) identification of condition for optimal conversion efficiency of layered structures, wherein the compatibility of optimal current density of individual phases is found to be the key; (3) extending the analysis of layered structures to the core-shell type of composites that are appropriate for many applications; (4) development of asymptotic analysis and homogenization of one-dimensional thermoelectric composites, for which closed-form solutions can be obtained; and (5) development of asymptotic analysis and homogenization of two- and three-dimensional thermoelectric composites, for which finite element simulations have been implemented. This set of theoretical and computational techniques can be used to guide the design and optimization of thermoelectric composites with optimal conversion efficiency.

\section{SUBJECT TERMS}

Homogenization, optimization, thermoelectric composite

\begin{tabular}{|c|c|c|}
\hline \multicolumn{3}{|c|}{ 16. SECURITY CLASSIFICATION OF: } \\
\hline a. REPORT & b. ABSTRACT & c. THIS PAGE \\
U & $\mathrm{U}$ & $\mathrm{U}$ \\
\hline
\end{tabular}

\section{LIMITATION OF ABSTRACT}

UU
10. SPONSOR/MONITOR'S ACRONYM(S)

AFOSR

11. SPONSOR/MONITOR'S REPORT NUMBER(S) 


\section{INSTRUCTIONS FOR COMPLETING SF 298}

1. REPORT DATE. Full publication date, including day, month, if available. Must cite at least the year and be Year 2000 compliant, e.g. 30-06-1998; xx-06-1998; xx-xx-1998.

2. REPORT TYPE. State the type of report, such as final, technical, interim, memorandum, master's thesis, progress, quarterly, research, special, group study, etc.

3. DATES COVERED. Indicate the time during which the work was performed and the report was written, e.g., Jun 1997 - Jun 1998; 1-10 Jun 1996; May - Nov 1998; Nov 1998.

4. TITLE. Enter title and subtitle with volume number and part number, if applicable. On classified documents, enter the title classification in parentheses.

5a. CONTRACT NUMBER. Enter all contract numbers as they appear in the report, e.g. F33615-86-C-5169.

5b. GRANT NUMBER. Enter all grant numbers as they appear in the report, e.g. AFOSR-82-1234.

5c. PROGRAM ELEMENT NUMBER. Enter all program element numbers as they appear in the report, e.g. $61101 \mathrm{~A}$.

5d. PROJECT NUMBER. Enter all project numbers as they appear in the report, e.g. 1F665702D1257; ILIR.

5e. TASK NUMBER. Enter all task numbers as they appear in the report, e.g. 05; RF0330201; T4112.

5f. WORK UNIT NUMBER. Enter all work unit numbers as they appear in the report, e.g. 001; AFAPL30480105.

6. AUTHOR(S). Enter name(s) of person(s) responsible for writing the report, performing the research, or credited with the content of the report. The form of entry is the last name, first name, middle initial, and additional qualifiers separated by commas, e.g. Smith, Richard, J, Jr.

\section{PERFORMING ORGANIZATION NAME(S) AND} ADDRESS(ES). Self-explanatory.

\section{PERFORMING ORGANIZATION REPORT NUMBER.} Enter all unique alphanumeric report numbers assigned by the performing organization, e.g. BRL-1234; AFWL-TR-85-4017-Vol-21-PT-2.

\section{SPONSORING/MONITORING AGENCY NAME(S)} AND ADDRESS(ES). Enter the name and address of the organization(s) financially responsible for and monitoring the work.

10. SPONSOR/MONITOR'S ACRONYM(S). Enter, if available, e.g. BRL, ARDEC, NADC.

\section{SPONSOR/MONITOR'S REPORT NUMBER(S).}

Enter report number as assigned by the sponsoring/ monitoring agency, if available, e.g. BRL-TR-829; -215.

12. DISTRIBUTION/AVAILABILITY STATEMENT. Use agency-mandated availability statements to indicate the public availability or distribution limitations of the report. If additional limitations/ restrictions or special markings are indicated, follow agency authorization procedures, e.g. RD/FRD, PROPIN, ITAR, etc. Include copyright information.

13. SUPPLEMENTARY NOTES. Enter information not included elsewhere such as: prepared in cooperation with; translation of; report supersedes; old edition number, etc.

14. ABSTRACT. A brief (approximately 200 words) factual summary of the most significant information.

15. SUBJECT TERMS. Key words or phrases identifying major concepts in the report.

16. SECURITY CLASSIFICATION. Enter security classification in accordance with security classification regulations, e.g. U, C, S, etc. If this form contains classified information, stamp classification level on the top and bottom of this page.

17. LIMITATION OF ABSTRACT. This block must be completed to assign a distribution limitation to the abstract. Enter UU (Unclassified Unlimited) or SAR (Same as Report). An entry in this block is necessary if the abstract is to be limited. 


\title{
The Homogenization and Optimization of Thermoelectric Composites
}

\author{
Final Report
}

Jiangyu Li

\begin{abstract}
We seek to understand the effective behavior of thermoelectric composites using rigorous homogenization technique in this project. In the last three years, our accomplishment includes: (1) rigorous analysis of thermoelectric field distribution and effective properties of layered thermoelectric structures, showing that their effective figure of merit is not bounded by the constituent phases, in contrast to the previous claim; (2) identification of condition for optimal conversion efficiency of layered structures, wherein the compatibility of optimal current density of individual phases is found to be the key; (3) extending the analysis of layered structures to the core-shell type of composites that are appropriate for many applications; (4) development of asymptotic analysis and homogenization of one-dimensional thermoelectric composites, for which closed-form solutions can be obtained; and (5) development of asymptotic analysis and homogenization of two- and three-dimensional thermoelectric composites, for which finite element simulations have been implemented. This set of theoretical and computational techniques can be used to guide the design and optimization of thermoelectric composites with optimal conversion efficiency.
\end{abstract}


We seek to understand the effective behavior of thermoelectric composites using rigorous homogenization technique in this project. In the last three years, our accomplishment includes:

1. Rigorous analysis of thermoelectric field distribution and effective properties of layered thermoelectric structures, showing that their effective figure of merit is not bounded by the constituent phases, in contrast to the previous claim.

In a well-known paper by Bergman and Levy published in Journal of Applied Physics in 1991, it was claimed that the effective figure of merit of a composite material is bounded by its constituent. This conclusion, however, was built on the linearized thermoelectric transport equations, which is not appropriate under large temperature difference for power generation operation. By using fully coupled nonlinear thermoelectric transport equation, we showed that this conclusion is incorrect, and a layered composite can achieve an effective figure of merit that is higher than its constituent phases. This work clarified a major misconception in the community. This paper was published in Journal of Applied Physics.

2. Identification of condition for optimal conversion efficiency of layered structures, wherein the compatibility of optimal current density of individual phases is found to be the key.

In the course of analysis, we noted that the effective figure of merit of composites is actually ill-defined, due to nonlinearity involved. This is a well-known issue for nonlinear composites. As such, we analyzed the conversion efficiency of composite structure directly, and found that the effective conversion efficiency of a composite can be higher than its constituent. Even more important, we found that each constituent phase has its own optimal current density for high conversion efficiency, and in order for the composite to have overall optimal conversion efficiency, the optimal current density of each phase needs to be compatible with each other. Built on this observation, we also derived a new upper bound on the effective conversion efficiency of layered composites. This paper was published in Applied Physics Letters.

3. Extending the analysis of layered structures to the core-shell type of composites that are appropriate for many applications.

We then extended our analysis of layered composite structure to radial geometry, with core-shell type of configuration. Such configuration is desirable for many applications involved in waste heat recovery, for example, for example exhaust heat from automobiles. The conclusion here is essentially similar to the layered structure. This paper was published in Acta Mechanica.

4. Development of asymptotic analysis and homogenization of one-dimensional thermoelectric composites, for which closed-form solutions can be obtained. 
Here, we analyzed one-dimensional periodic thermoelectric composites using asymptotic analysis. Using separation of length scale and continuity of current density, we showed that zero-order term is the homogenized field distribution we are looking for, wherein the small scale fluctuation is smeared out when the size of heterogeneity approaches zero. Furthermore, we identified unit cell problems, on which the field distribution can be solved, and the governing equation for the homogenized field distribution can be obtained. These homogenized thermoelectric governing equations are more complicated than homogenous ones, but can still be solved analytically. This paper was published in Journal of Mechanics and Physics of Solids.

5. Development of asymptotic analysis and homogenization of two- and three-dimensional thermoelectric composites, for which finite element simulations have been implemented.

The asymptotic analysis was extended to two- and three-dimensional periodic composites, wherein the current density is no longer a constant due to higher dimensionality. This complicates the analysis substantially, nevertheless, we were able to show that the zero-order term is still the homogenized solution, and we were able to identify the unit cell problem as well. The solution to the unit cell problem, however, has to be attacked numerically using finite element method, which we developed with our own code. This allows us to calculate the effective thermoelectric properties of threedimensional composites with arbitrary microstructures. This work was published in another Journal of Mechanics and Physics of Solids paper.

In summary, we have developed a set of theoretical and computational techniques to analyze the effective behavior of thermoelectric composites, and they can be used to guide the design and optimization of thermoelectric composites with optimal conversion efficiency. 
1.

\section{Report Type}

Final Report

\section{Primary Contact E-mail}

Contact email if there is a problem with the report.

jjli@uw.edu

Primary Contact Phone Number

Contact phone number if there is a problem with the report

$1-206-543-6226$

Organization / Institution name

University of Washington

\section{Grant/Contract Title}

The full title of the funded effort.

The Homogenization and Optimization of Thermoelectric Composites

\section{Grant/Contract Number}

AFOSR assigned control number. It must begin with "FA9550" or "F49620" or "FA2386".

FA9550-12-1-0325

\section{Principal Investigator Name}

The full name of the principal investigator on the grant or contract.

Jiangyu Li

Program Manager

The AFOSR Program Manager currently assigned to the award

Arje Nachman

\section{Reporting Period Start Date}

$07 / 01 / 2012$

\section{Reporting Period End Date}

$12 / 31 / 2014$

\section{Abstract}

We seek to understand the effective behavior of thermoelectric composites using rigorous homogenization technique in this project. In the last three years, our accomplishment includes: (1) rigorous analysis of thermoelectric field distribution and effective properties of layered thermoelectric structures, showing that their effective figure of merit is not bounded by the constituent phases, in contrast to the previous claim; (2) identification of condition for optimal conversion efficiency of layered structures, wherein the compatibility of optimal current density of individual phases is found to be the key; (3) extending the analysis of layered structures to the core-shell type of composites that are appropriate for many applications; (4) development of asymptotic analysis and homogenization of onedimensional thermoelectric composites, for which closed-form solutions can be obtained; and (5) development of asymptotic analysis and homogenization of two- and threedimensional thermoelectric composites, for which finite element simulations have been implemented. This set of theoretical and computational techniques can be used to guide the design and optimization of thermoelectric composites with optimal conversion efficiency. 
Distribution Statement

This is block 12 on the SF298 form.

Distribution A - Approved for Public Release

\section{Explanation for Distribution Statement}

If this is not approved for public release, please provide a short explanation. E.g., contains proprietary information.

\section{SF298 Form}

Please attach your SF298 form. A blank SF298 can be found here. Please do not password protect or secure the PDF The maximum file size for an SF298 is $50 \mathrm{MB}$.

AFD-070820-035-Li.pdf

Upload the Report Document. File must be a PDF. Please do not password protect or secure the PDF . The maximum file size for the Report Document is 50MB.

\section{TE AFOSR_Final.pdf}

Upload a Report Document, if any. The maximum file size for the Report Document is 50MB.

Archival Publications (published) during reporting period:

Yang, Y., Xie, S. H., Ma, F. Y., \& Li, J. Y. (2012). On the effective thermoelectric properties of layered heterogeneous medium. Journal of Applied Physics, 111(1), 013510.

Yang, Y., Ma, F. Y., Lei, C. H., Liu, Y. Y., \& Li, J. Y. (2013). Is thermoelectric conversion efficiency of a composite bounded by its constituents?. Applied Physics Letters, 102(5), 053905.

Yang, Y., Ma, F. Y., Lei, C. H., Liu, Y. Y., \& Li, J. Y. (2013). Nonlinear asymptotic homogenization and the effective behavior of layered thermoelectric composites. Journal of the Mechanics and Physics of Solids, 61(8), 1768-1783.

Yang, Y., Gao, C., \& Li, J. (2014). The effective thermoelectric properties of core-shell composites. Acta Mechanica, 225(4-5), 1211-1222.

Yang, Y., Lei, C., Gao, C. F., \& Li, J. (2015). Asymptotic homogenization of threedimensional thermoelectric composites. Journal of the Mechanics and Physics of Solids, 76, 98-126.

Changes in research objectives (if any):

Change in AFOSR Program Manager, if any:

Extensions granted or milestones slipped, if any:

\section{AFOSR LRIR Number}

\section{LRIR Title}

Reporting Period

Laboratory Task Manager

Program Officer

Research Objectives

Technical Summary

Funding Summary by Cost Category (by FY, \$K) 


\begin{tabular}{|l|l|l|l|}
\hline & Starting FY & $\mathrm{FY}+1$ & $\mathrm{FY}+2$ \\
\hline Salary & & & \\
\hline Equipment/Facilities & & & \\
\hline Supplies & & & \\
\hline Total & & & \\
\hline
\end{tabular}

\section{Report Document}

Report Document - Text Analysis

Report Document - Text Analysis

Appendix Documents

2. Thank You

E-mail user

Apr 13, 2015 02:32:24 Success: Email Sent to: jjli@uw.edu 